\title{
Zusatznutzen für herzkranke Diabetiker
}

_ Bei Diabetikern mit kardiovaskulären Erkrankungen wurde der SGLT2-Inhibitor Empagliflozin bisher zur Verbesserung der Blutzuckerkontrolle eingesetzt. Nun kann er auch zur Reduktion der kardiovaskulären Sterblichkeit verordnet werden. Grundlage der Indikationserweiterung durch die europäische $\mathrm{Zu}$ -

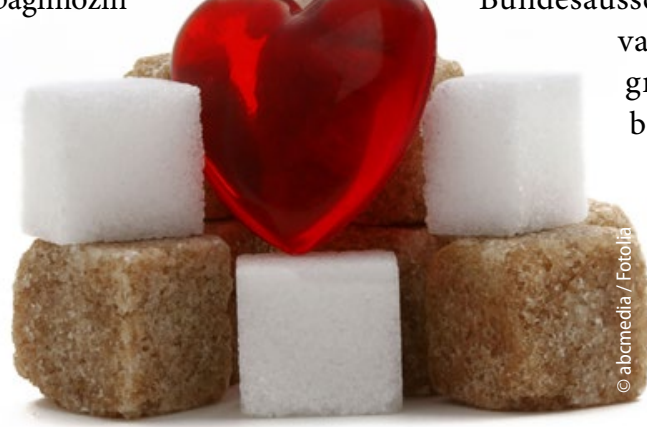

Erhöhte Blutzuckerwerte bedrohen das Herz.
Aufgrund dieser Ergebnisse räumte der Gemeinsame Bundesausschuss für relevante Patientengruppen einen beträchtlichen

Zusatznutzen ein, und zwar bei Kombination mit Metformin, mit einem oder zwei anderen oralen Antilassungsbehörde waren Ergebnisse der EMPA-REG-OUTCOME-Studie [Zinman B et al. N Engl J Med. 2015;373: 2117-28].

Die Studie mit ca. 7.000 langjährigen Diabetikern $\left(\mathrm{HbA}_{1 \mathrm{c}}\right.$-Wert im Schnitt: 8,1\%) und kardiovaskulären Erkrankungen brachte ein "fulminantes Ergebnis“, berichtete Prof. Christian Schneider, Köln. Die Therapie mit Empagliflozin (Jardiance ${ }^{\varpi}$ ) - zusätzlich zu einer optimalen Therapie von Diabetes und Herzerkrankung - reduzierte das relative Risiko für den primären Endpunkt (kardiovaskulärer Tod, Herzinfarkt, Insult) nach 3,1 Jahren um $14 \%$ (10,4\% vs. $12,1 \%)$. Das relative Risiko für kardiovaskulären Tod wurde um $38 \%$ (3,7\% vs. 5,9\%) reduziert, für Tod jeder Ursache um $32 \%$ (5,7\% vs. $8,3 \%)$ und für Hospitalisierungen wegen Herzinsuffizienz um $35 \%$ (2,7\% vs. $4,1 \%$ ). Gleichzeitig blieb die Nierenfunktion unter Empagliflozin länger und besser erhalten als unter Placebo, berichtete Prof. Baptist Gallwitz, Tübingen. In Deutschland gilt der Einsatz bei die„Die Therapie ist einfach, nicht teuer, sie bringt kein Risiko, keine Regressgefahr, kommt ohne Schulungsbedarf aus, und sie verbessert die Lebenserwartung deutlich. Ich stelle meine Patienten konsequent um", so Schneider.

„Es ist das erste Medikament in der Diabetologie mit beträchtlichem Zusatznutzen“, ergänzte Gallwitz. Bei einer $\mathrm{GFR}<45 \mathrm{ml} / \mathrm{min} / 1,73 \mathrm{~m}^{2}$ müsse man es absetzen, sonst gebe es keine Kontraindikationen. Einzige Nebenwirkungen, über die der Patient aufgeklärt werden sollte, sind Genitalinfektionen in knapp 5\% der Fälle.

Dr. Dirk Einecke

- Presseveranstaltung "Kardiovaskuläre Risikoreduktion in der Therapie des Typ-2-Diabetes: Regulatorisches Update zu Jardiance ${ }^{\varpi \prime ; ~ F r a n k f u r t ~ a . ~ M ., ~ F e b r u a r ~} 2017$ (Veranstalter: Boehringer Ingelheim und Lilly)

\section{Neues Medikament gegen periodische Fiebersyndrome}

Periodische Fiebersyndrome (PFS) treten meist bereits im Kindesalter auf und verursachen wiederkehrende Fieberschübe mit potenziell lebensbedrohlichen Komplikationen. PFS sind genetisch bedingt und beruhen auf einer Störung des Immunsystems. Am 23. Februar 2017 erteilte die EU-Kommission die Zulassung für Canakinumab (Ilaris ${ }^{\circledast}$ ) für die Therapie des Familiären Mittelmeerfiebers (FMF), des Hyperimmunglobulin-D-Syndroms (HIDS) und des Tumornekrosefaktor-Rezeptor-assoziierten periodischen Syndroms (TRAPS).

Red.

- Nach Informationen von Novartis

\section{Einstellung der Produktion von Strontiumranelat}

Servier stellt ab 31. August 2017 weltweit die Produktion und den Vertrieb von Protelos ${ }^{\circledR}$ (Strontiumranelat $2 \mathrm{~g}$ ) ein. Die Firma informiert bereits jetzt, um den Ärzten genügend Zeit zu geben, die mit Protelos ${ }^{\circledR}$ behandelten Patienten auf andere Medikamente umstellen zu können. 